\title{
Biosensor Interface: Interactive Media Art Using Biometric Data
}

\author{
Dongjo Kim and Hyunggi Kim \\ Dept. of advanced image Graduate School of Advanced Imaging Science, \\ Multimedia and Film, Chung-Ang University \\ sojudj@gmail.com,unzi@cau.ac.kr
}

\begin{abstract}
Recently, as biomimetics is applied to design and engineering and receive attention from the society, its artistic value also is receiving people's interest. Data visualization is expressed regarding brain wave, iris, fingerprint, pulse, body fat, etc. Particularly, developing of convergence contents, biometric data is also influencing development of affective data. This study is focused on research on media art using biometric data, and an artwork using pulse data was created as an example based on review of relevant research. By developing the process in which the audience sees their real-time biometric data, aesthetic contemplation according to sophistication of emotional contents will be studied.
\end{abstract}

Keywords: Bio art, Biometric Data, Interactive art, HRM(Heart Rate Monitor), Ring Sensor, Data Visualization

\section{Introduction}

Human biometric data is researched for various applications. Design of computer control based on brain wave and security system based on fingerprint and iris recognition, emotional treatment system using the heartbeat, and healthcare service using various biorhythms are examples of directly using the data created from humans. Recently, biometric data collected from bio sensors are applied to interactive art as well. From the psychological viewpoint, realtime expression and feedback of biometric data are very important because they can help selfrecognition and expansion of communication based on understanding of one's own emotion and body. Humans express emotion based on five senses but they also use overall functions of the body to communicate with one another. The feelings toward an artwork are communicated in a multi-dimensional way, and the process of sending and receiving biometric data with everything is repeated. Reaction of human body, which is assumed to be biometric data, including the body temperature, blood pressure, pulse, pulse rate, blood, water, and salt, is not consistent throughout the day, but slightly varies depending on the environment in which it operates with biometric data. Accordingly, human body generates physical, intellectual, and emotional rhythms. In this paper, an artwork that used biometric data collected through sensors was studied. A subject's pulse data is expressed through an object in real time and he or she views it as an artwork as an audience. For this process, interactive art acts as a medium and, naturally, the audience form relationship with the artwork and learns the intent of the artist. Technological development and convergence of different fields create diverse contents and develop artworks to which human emotion is applied. This study will discuss how using biometric technology can improve our understanding of emotion and continuous research and artistic expression. 


\section{Related work}

Currently, in developing affective contents, the most actively researched biometric data is brain wave. Neocomimi of Neurowear, which was created as an artwork and recently commercialized, was made with cat's ears as a motif. It measures the audience's brain wave and the sensors, which is like a headband, has cat ears, which move according to the level of brain wave. The ears stand when the person focuses, and lie down when relaxed, and, if the person focuses and relaxes at the same time, the ears dance. The objet's movement can be controlled by controlling the brain wave after putting on the gear. This project used brain wave as a new communication tool to expand the body and ability of humans.

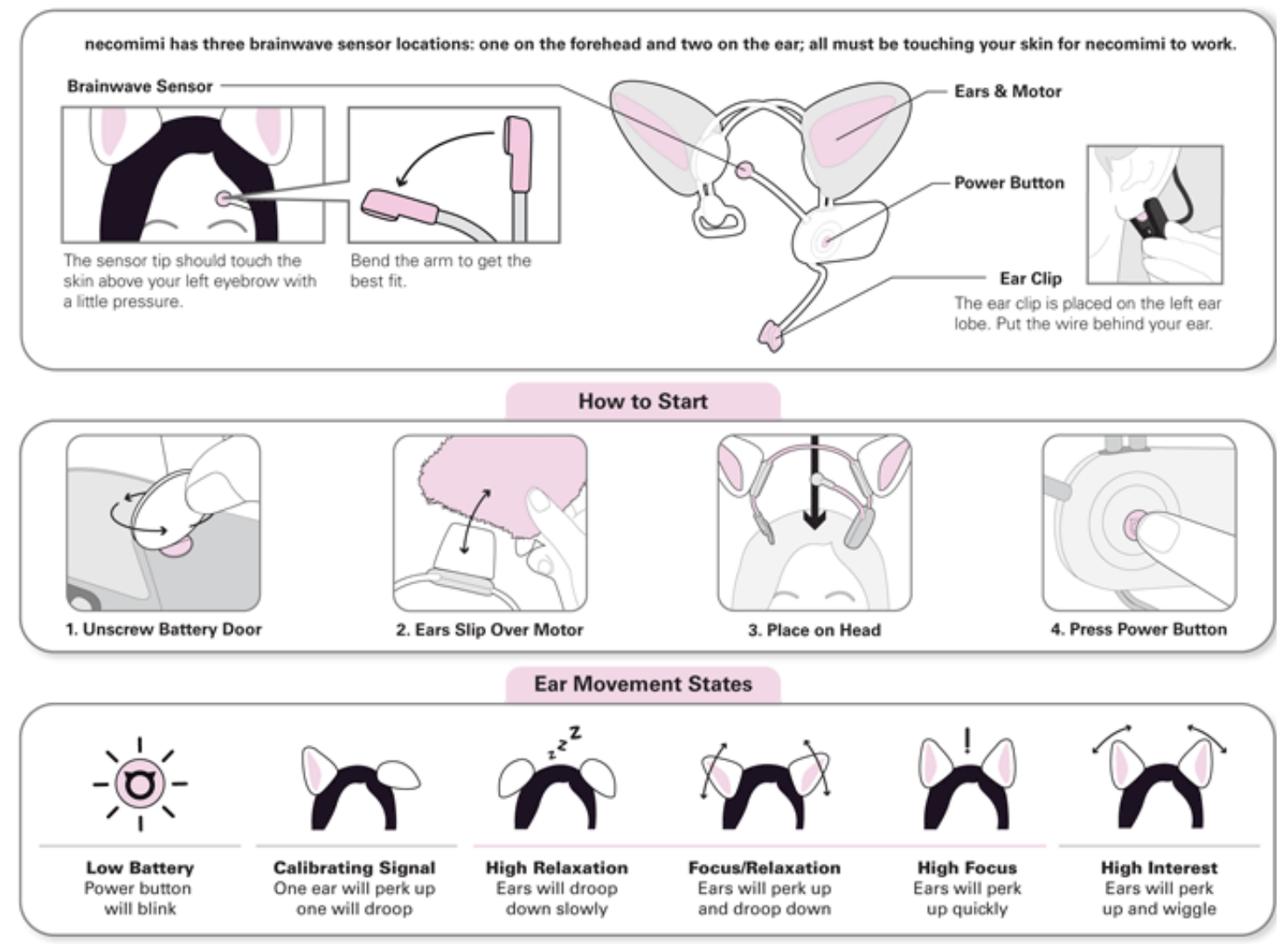

Figure 1. Instructions for Use

Figure 2 is Rafael Lozano-Hemmer's Pulse Room, which was shown in Plataforma in 2006. It is made of total 300 incandescent lamps and installed on the ceiling at $3 \mathrm{~m}$ by using a cable. On one side, the interface measures the pulse, and when the audience is holding it, the measured heart rate is expressed by the closes lamp's flickering, and then one of the incandescent lamps in the overall space. This process is repeated and when the number of audience increases, the 300 lamps flicker as if they are individually alive. 


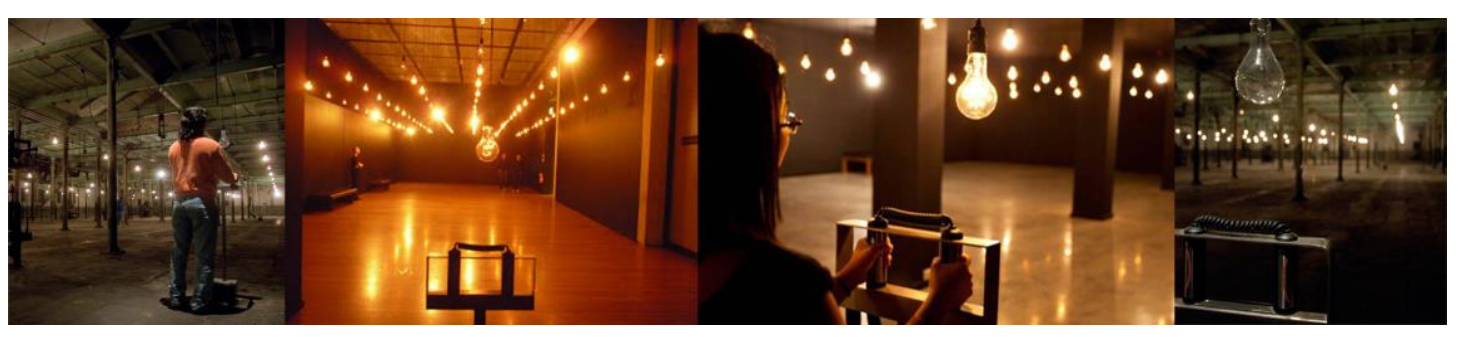

Figure 2. Rafael Lozano-Hemmer's Pulse Room

Figure 3 is George Zisiadis's Pulse of the city, which was first exhibited in Urban Prototyping Festival in San Francisco in October 2012, and now in five areas of Boston streets as an interactive public art installation. It is made in a heart shape, and when the viewer holds the handle on the either side, it immediately detects and checks the heart rate in real time for one minute, which is then used as a beat to make music that will be played through the built-in speaker. In the confusing noise of the city, pedestrians listen to their biometric data and are entertained by it.

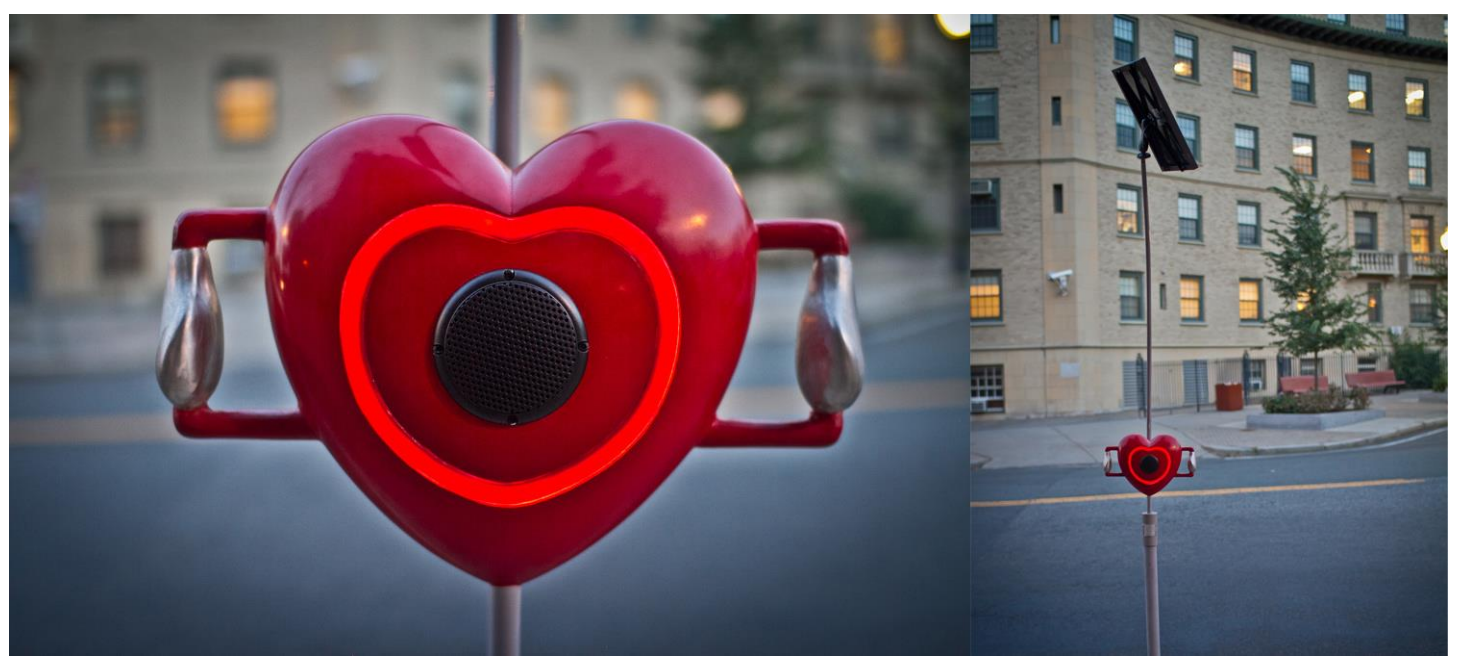

Figure 3. George Zisiadis's Pulse of the city

\section{Research of Interactive Artwork}

\subsection{Concept and functional}

The author used pulse, among biometric data, of the audience to create an interactive artwork. The data generated from the audience was used as a medium to facilitate communication of the audience, artwork, and artist, and it is repeatedly converted and played to the audience. The audience, who changes all the time, focuses on their body and the objet that is expressed according to the change. The audience and artwork share the same biorhythm and, in the process, communicate with each other. The expression was based on visualization and sonification. Visualization mainly affected flickering of the light while sonification designated the start of the play cycle of the built-in sound. Palpitation and bud, which used pulse, was made in two versions: one that generates one reaction - light, sound, or movement - and one that expresses combination of factors. For Ver. 1, a round and flat objet 
is laid down and when the audience puts the ring-type pulse sensor on their finger and the pulse data, which is measured real-time, makes the light dim as if it is breathing. Secondly, the data is received from the audience by the same process, and the black fabric moves up and down like a heart. Third, recorded heart beat is played at the starting point of the pulse data. These three types of artwork were exhibited as a prototype, and the audience was entertained to see their pulse expressed in real time and closely observed and even tried to control the continuous and slight changes. Ver. 2 is a developed form of Ver. 1, and made of a bud and long stem. The audience easily discovered the position of the sensor, which was attached at the end of the stem. The pulse is measured when the audience puts their finger into the tip of the stem, and, after one or two seconds, the bud starts dimming like it is alive and breathing. And the speaker built in the pot section uses the entire pot as a sound box and plays the heart beat at the starting point of the pulse data. The artwork based on visualization and sonification of the audience's pulse data increased the audience's focus and participation. The two versions used pulse of the audience to breathe life into the artwork. As its motif was babies sleeping sound in their mother's arms, it can be used as a therapy combining image and music.

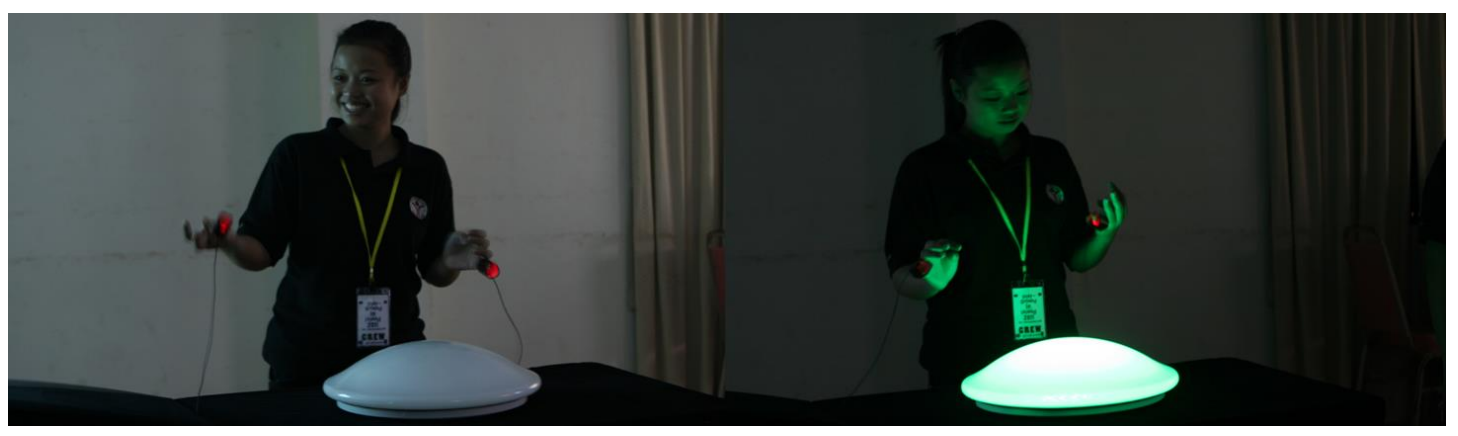

Figure 4. Palpitation and bud Ver.1

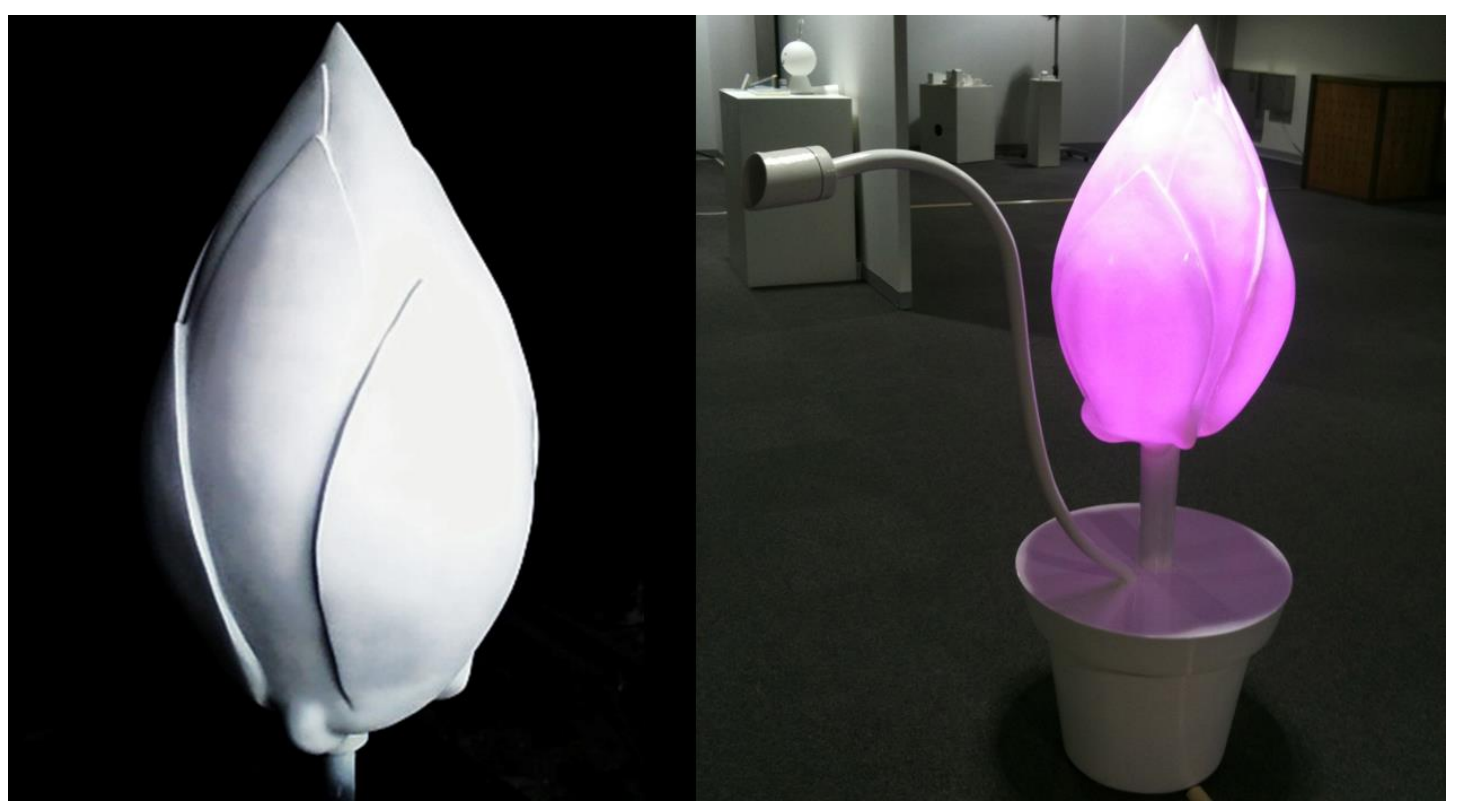

Figure 5. Palpitation and bud Ver.2 


\subsection{Hardware}

It looks like a bud as a symbol of the beginning, and is divided into the bud, pot, and stem, by their function. The bud section is made of 100 LEDs and transparent FRP, and the pot section has the main controller, speaker, battery, and power device. Lastly, at the end of the long stem is the sensor. The sensor used for measuring pulse is RP320 of Laxtha and a ringtype pulse wave sensor, which is an active sensor that can detect pulse wave with a continuum method. The photo-diode arranged to face the finger is designed to detect pulse wave by reflection. In other words, photoelectric plethysmo-graphy (PPG) signal was used. To observe conventional pulse waveform, a circuit that differentially amplifies and filters sensor output signals was added for reversal of polarity. Figure 6 is a block diagram of the ring-type pulse sensor.

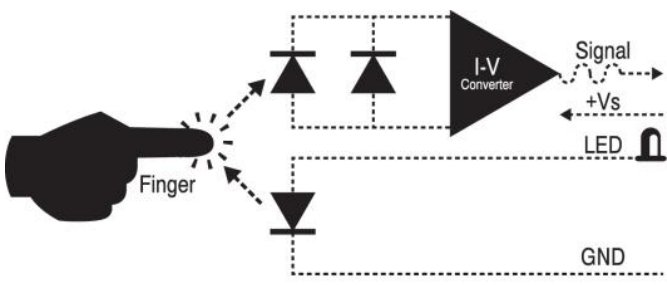

Figure 6. Block Digram of the Ringtype pulse sensor

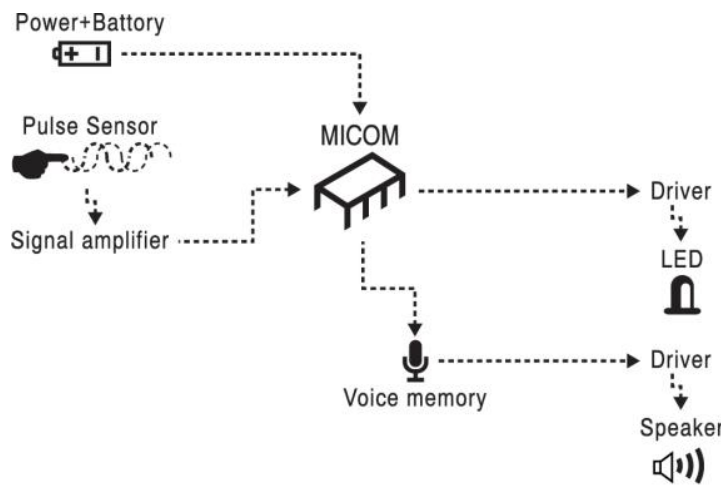

Figure 7. block Digram of palpitation and bud Ver.2

\section{Conclusion}

Recently, with development of ubiquitous health care service, big data of biometric data and its importance are often discussed. Technological development is expected to expand convergence healthcare industries as well. Today, humans wish to properly know and understand their body. Even in the art field, there are new words like bio-art and biomimetics art, which shows recognition of its aesthetic value and it allows exhibition accustomed to the audience. In this paper, interactive media artworks using biometric data were reviewed and artwork production was studied. When making the prototype, Ver. 1, only one output mode was used to give feedback to the audience, while Ver. 2 used feedback to the biometric data was outputted in combined forms. The findings indicated using biometric data increases pleasure and focus of the audience, and that combined forms bring out more participation from the audience. Feedback of the artwork, which is communicated through intuitive expansion of body, causes instant interest like replication but cannot increase pleasure and focus innate to the artwork. On the other hand, communication of the artwork using combined feedback maximizes affective data of the artwork perceived by the audience, and expands visible focus and entertainment of the artwork. Based on the findings, the author would like to make various attempts to integrate multimodal biometric data and express the segmented and complex forms of feedback to the audience. Furthermore, technology must be developed for efficient collection of biometric data, which is planned as the follow-up study. The author 
hopes that more artists will develop affective contents based on biometric data to share more enriched artistic value.

\section{References}

[1] E. -s. Song and H. Rhee, "A Study on bio-sensing systems for interactive media arts", Journal of digital design, vol. 13 no. 1, (2013), pp. 25-33.

[2] A. Dix, J. E. Finlay, G. D. Abowd and R. Beale, "Human-Computer Interaction", Prentice Hall, (2003).

[3] A. Valli, "The Design of Natural Interaction", Multimedia Tools and Applications, vol. 38, no. 3, (2008), pp. 295-305.

[4] J. H. Oh and C. -K. Shi, "Interactive Art in the Age of Digital Reproduction", HCI International, vol. 374, (2013), pp. 287-291.

[5] G. Tina, "Empathy and interactivity: Creating emotionally empathic circuits between audiences and interactive arts", Computers in Entertainment, vol. 8 no. 1, (2010).

[6] R. Klich, "The 'unfinished' subject: Pedagogy and performance in the company of copies, robots, mutants and cyborgs", International Journal of Performance Arts and Digital Media, vol. 8, no. 2, (2012), pp. 155170.

[7] N. De Liso, "From mechanical arts to the philosophy of technology", Economics of Innovation and New Technology, vol. 22, no. 7, (2013), pp. 726-750.

[8] C. Pirson, "New anatomical shows: The body of the artist in the bio art", Degrés, Issue 151-152, (2012), pp. i1 - i17.

[9] H. Kim, H. -j. Lee and E. Y. -1. Do, "Drawing together: Exploring collaborative user engagement in art exhibitions", HCI International 2011, vol. 10, LNCS6770, (2011), pp. 142-151 Springer, Heidelberg.

[10] J. H. Oh and C. -K. Shi, "Interactive Art in the Age of Digital Reproduction", HCI International, vol. 374, (2013), pp. 287-291.

[11] J. Hu, D. Le, M. Funk, F. Wang and M. Rauterberg, "Attractiveness of an interactive public art installation", Lecture Notes in Computer Science, 8028 LNCS, (2013), pp. 430-438.

[12] M. Donnarumma, "Hypo Chrysos: Mapping in interactive action art using bioacoustics sensing", International Conference on Tangible, Embedded and Embodied Interaction, vol. 7, (2013), pp. 383-385.

[13] A. G. Forbes, T. Höllerery and G. Legradyz, "Generative fluid profiles for interactive media arts projects", Computational Aesthetics, (2013), pp. 37-44.

[14] P. Walters, D. McGoran and I. Ieropoulos, "Digital fabrication of a novel bio-actuator for bio-robotic art and design", International Conference on Digital Printing Technologies, (2011), pp. 496-499.

[15] D. Kim and H. Kim, "Bio-Sensing: pulse sensing for interactive media art", Workshop on Games and Graphics, (2013) Second, Jeju National University.

[16] LAXTHA Inc. http://www.laxtha.com.

[17] neurowear Inc. http://neurowear.com.

[18] http://www.necomimi.com/.

[19] http://www.lozano-hemmer.com/.

[20] http://www.georgezisiadis.com/.

\section{Authors}

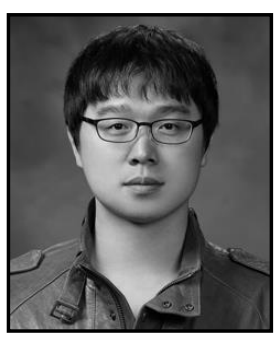

\section{Dongjo Kim}

ABD, Department of advanced image Graduate School of Advanced Imaging Science, Multimedia and Film, Chung-Ang University 


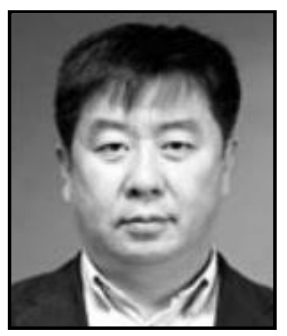

\section{Hyunggi Kim}

Professor, Department of advanced image Graduate School of Advanced Imaging Science, Multimedia and Film, Chung-Ang University 
International Journal of Bio-Science and Bio-Technology Vol.6, No.1 (2014) 